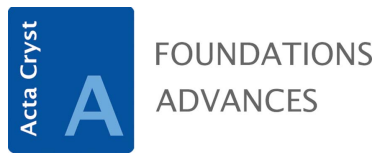

ISSN 2053-2733
Keywords: book review; history of crystallography; Nobel Prize.

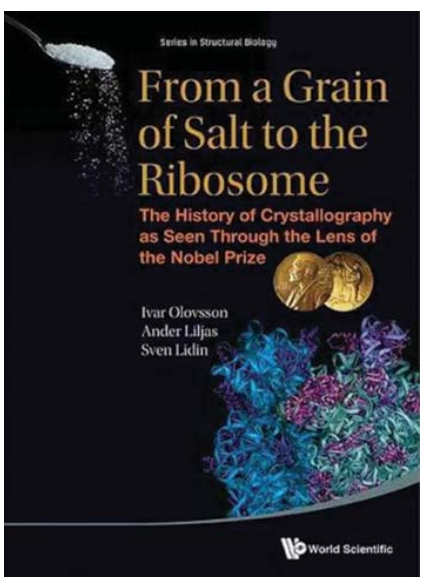

(C) 2015 International Union of Crystallography

\section{From a Grain of Salt to the Ribosome. The History of Crystallography as Seen Through the Lens of the Nobel Prize. Series in Structural Biology, Volume 4, edited by Ivar Olovsson, Anders Liljas and Sven Lidin. World Scientific, 2014. Pp. 536. Price GBP 98.00. ISBN 978-981-4623-11-7.}

\author{
Davide Viterbo*
}

Dipartimento di Scienze e Tecnologie Avanzate, Università del Piemonte Orientale, Viale T. Michel 11, I-15121 Alessandria, Italy. *Correspondence e-mail: davide.viterbo@mfn.unipmn.it

The publication of From a Grain of Salt to the Ribosome comes just after the intense promotional activities of 2014, which was declared by the United Nations as the International Year of Crystallography, to remind us that we must keep promoting our science, and provides a lively historical account of the impact that crystallography has had on many branches of science. It is certainly not accidental that the three Swedish authors have chosen to tell us about the successes of crystallography through the lens of the Nobel Prize. From the first prize in 1901 to Roentgen, to the one awarded to Kobilka and Lefkowitz in 2012, a total of 21 prizes have had crystallography at their centre.

The book is the result of a work of detailed historical documentation (proved by the exhaustive list of references and the selection of reprints), for which we must be grateful to the authors, who have been able to elaborate this large mass of information into a pleasant book full of relevant and interesting stories.

After a short introduction, with a brief history of crystallography, the first part of the book is formed by 21 short chapters, each containing relevant information on the motivations, the awardees and the relevance of one of the following 21 selected Nobel Prizes:

1901 Physics - W. C. Roentgen: in recognition of the extraordinary services he has rendered by the discovery of the remarkable rays subsequently named after him.

1914 Physics - M. von Laue: for his discovery of the diffraction of X-rays by crystals.

1915 Physics - W. H. and W. L. Bragg: for their services in the analysis of crystal structure by means of X-rays.

1937 Physics - C. J. Davisson and G. P. Thomson: for their experimental discovery of the diffraction of electrons by crystals.

1946 Chemistry - J. B. Sumner (with J. H. Northrop and W. M. Stanley): for his discovery that enzymes can be crystallized.

1954 Chemistry - L. C. Pauling: for his research on the nature of the chemical bond and its application to the elucidation of the structure of complex substances.

1962 Medicine - F. Crick, J. Watson and M. Wilkins: for their discoveries concerning the molecular structure of nucleic acids and its significance for information transfer in living material.

1962 Chemistry - M. F. Perutz and J. C. Kendrew: for their studies of the structures of globular proteins.

1964 Chemistry - D. Crowfoot Hodgkin: for her determination by X-ray techniques of the structures of important biochemical substances.

1976 Chemistry - W. N. Lipscomb: for his studies on the structure of boranes illuminating problems of chemical bonding.

1982 Chemistry - A. Klug: for his development of crystallographic electron microscopy and his elucidation of biologically important nucleic acid-protein complexes.

1985 Chemistry - H. A. Hauptman and J. Karle: for their outstanding achievements in the development of direct methods for the determination of crystal structures. 
1988 Chemistry - J. Deisenhofer, R. Huber and H. Michel: for the determination of the three-dimensional structure of a photosynthetic reaction centre.

1994 Physics - B. N. Brockhouse and C. G. Shull: for pioneering contributions to the development of neutronscattering techniques for studies of condensed matter.

1997 Chemistry - P. Boyer, J. E. Walker and J. C. Skou: for their elucidation of the enzymatic mechanism underlying the synthesis of adenosine triphosphate (ATP).

2003 Chemistry - (P. Agre and) R. MacKinnon: for discoveries concerning channels in cell membranes, for structural and mechanistic studies of ion channels.

2006 Chemistry - R. D. Kornberg: for his studies of the molecular basis of eukaryotic transcription.

2009 Chemistry - V. Ramakrishnan, T. Stetz and A. Yonath: for studies of the structure and function of the ribosome.

2011 Chemistry - D. Shechtman: for the discovery of quasicrystals.

2012 Chemistry - B. K. Kobilka (and J. Lefkowitz): for studies of G-protein-coupled receptors.

The scheme of these chapters is simple and essential, while maintaining the relevant information: a short biography of each recipient (only those who have a closer relation to crystallography are considered) is followed by an illustration of the content, significance and consequences of the discoveries and completed by a number of relevant references. This common scheme does not render the sequence of chapters monotonous to read, for the authors have enlivened each chapter with a number of stories and anecdotes on the lives of most awardees and on the route to their nomination. The result is a very easy and pleasant part of the book that can be read as a novel and not just as a good reference to be consulted when needed.
The second part of the book contains a well selected and ample set of reprints of the main papers describing the results that inspired the nomination of the awardees of the prizes. After the papers by Roentgen and Laue in Annalen der Physik und Chemie and by Bragg in Proceedings of the Royal Society of London, I would like to mention the papers on DNA by Watson, Crick, Wilkins and Franklin and those on myoglobin and hemoglobin by Perutz and Kendrew in Nature, the papers by Hauptman and Karle in Acta Crystallographica, those on the ribosome by Yonath, Ramakrishnan and Steitz in Biophysical Chemistry and Science, and the paper on quasicrystals by Shechtman from Physical Review Letters. The purpose of this collection of reprints is to give the reader the possibility of consulting the original papers and gaining a full understanding of the achievements made in science through crystallography, directly from their authors.

In its basic conception the book, based on the illustration of such a large number of Nobel Prizes, certainly extols crystallography for its merits and, being written by three scientists, may be criticized by historians for not being historically objective, but Nobel Prizes are not a myth, and some embellishment can not contradict this fact. After all, we love our science and have to promote it!

This book provides a vivid insight into the development of crystallography, and is certainly a valid tool for promoting our science and for showing our chemist, physicist, mineralogist, biologist and material scientist 'users' that we are constantly improving our tools in order to obtain a clearer picture of the structure of matter even in the most complicated cases: crystallography is ready to face the coming 100 years of progress and continue its essential role in science and life! 